\title{
CONVEXITY OF THE IDEAL BOUNDARY FOR COMPLETE OPEN SURFACES
}

\author{
JIN-WHAN YIM
}

\begin{abstract}
For complete open surfaces admitting total curvature, we define several kinds of convexity for the ideal boundary, and provide examples of each of them. We also prove that a surface with most strongly convex ideal boundary is in fact a generalization of a Hadamard manifold in the sense that the ideal boundary consists entirely of Busemann functions.
\end{abstract}

\section{INTRODUCTION}

Let $M$ be a complete, noncompact, connected, and oriented Riemannian manifold of dimension 2. The total curvature of $M$ is defined by the improper integral $\int_{M} K_{M}$ of the Gaussian curvature $K_{M}$ over $M$. A well-known result of Cohn-Vossen [3] states that if the total curvature exists, then

$$
\int_{M} K_{M} \leq 2 \pi \chi(M),
$$

where $\chi(M)$ is the Euler characteristic of $M$.

Since the total curvature of noncompact $M$ is not a topological invariant but depends on Riemannian metric, it should describe certain properties of the geometric structure of $M$. This idea motivated many works. Especially, the difference, $2 \pi \chi(M)-\int_{M} K_{M}$, has been studied in connection with the asymptotic behavior of the rays (see [8], [10]).

On the other hand, if $M$ is a compact oriented surface with boundary, then by the Gauss-Bonnet theorem the value $2 \pi \chi(M)-\int_{M} K_{M}$ can be interpreted as the convexity of the boundary. Therefore, together with Cohn-Vossen's result, this suggests that an open surface has in a sense convex boundary at infinity when it is suitably defined. This boundary at infinity is called "the ideal boundary" of $M$. In this paper, we will study the notion of ideal boundary, and introduce the concept of convexity at infinity of a complete open surface admitting total curvature.

The ideal boundary of an open manifold can be defined in several ways. We will follow the lead of [1], [6]. Let $M$ be a noncompact complete Riemannian manifold with distance function $d($,$) . For each x \in M$ let $d_{x}$ be the continuous function $y \rightarrow d(x, y)$. The map $x \rightarrow d_{x}$ defines an embedding of $M$ into

Received by the editors July 21,1993 and, in revised form, October 26, 1993; originally communicated to the Proceedings of the AMS by Christopher Croke.

1991 Mathematics Subject Classification. Primary 53C20, 53C45.

This research was partially supported by GARC-KOSEF. 
$C(M)$, the set of continuous functions on $M$ with the topology of uniform convergence on compact sets. We consider $C_{*}(M) \stackrel{\text { def }}{=} C(M)$ (constant functions) with the induced topology and the induced embedding $i: M \rightarrow C_{*}(M)$ defined by $i(x)=\bar{d}_{x}$, the equivalence class of $d_{x}$. The boundary $\partial M$ is defined as $C l(M)-i(M)$, where $C l(M)$ is the closure of $i(M)$ in $C_{*}(M)$. A point in $\partial M$ is an equivalence class of functions called horofunctions, which are well defined up to a constant.

There are other ways to define a space at infinity. A unit speed geodesic $\gamma:[0, \infty) \rightarrow M$ is called a ray if $d(\gamma(s), \gamma(t))=|s-t|$ for any $s, t \in[0, \infty)$. For any $p \in M$ let $\left\{q_{i}\right\}$ be a divergent sequence of points in $M$, and for each $i$ let $\gamma_{i}$ be a unit speed minimal geodesic from $p$ to $q_{i}$. Then the sequence $\left\{\gamma_{i}\right\}$ has a convergent subsequence and the limit is a ray emanating from $p$. Therefore an open manifold has rays emanating from every point, and the boundary $\partial M$ can be defined as equivalence classes of these rays. For Hadamard manifolds, an asymptotic relation of rays is known to define the ideal boundary equivalent to the first one we described. In this case, two rays $\gamma_{1}$ and $\gamma_{2}$ are called asymptotic if there is a constant $\alpha$ such that $d\left(\gamma_{1}(t), \gamma_{2}(t)\right) \leq \alpha$ for every $t \geq 0$. In fact two asymptotic rays define the same horofunction called the Busemann-function up to a constant. For a ray $\gamma:[0, \infty) \rightarrow M$ the Busemann-function $b_{\gamma}$ of $\gamma$ is defined by

$$
b_{\gamma}(x)=\lim _{t \rightarrow \infty}(d(x, \gamma(t))-t) .
$$

For a Hadamard manifold, it is known that all horofunctions are Busemannfunctions, and therefore the ideal boundary can be defined by the set of Busemann-functions or the equivalence classes of rays representing the same Busemann-functions up to constants.

These beautiful properties of Hadamard manifolds however fall apart in the general case. Even for a surface, if it is not a Hadamard manifold, a horofunction may not be a Busemann-function. We will give an example of a non-Busemann horofunction, and will state a sufficient condition when all horofuctions are Busemann-functions, which is a generalization of the Hadamard manifold case in dimension 2 .

A ray $\sigma:[0, \infty) \rightarrow M$ is said to be asymptotic to a ray $\gamma:[0, \infty) \rightarrow M$ if there exist a monotone sequence $\left\{t_{n}\right\}$ of positive numbers such that $t_{n} \rightarrow \infty$ as $n \rightarrow \infty$ and a sequence $\left\{\sigma_{n}:\left[0, \ell_{n}\right] \rightarrow M\right\}$ of minimal geodesics such that $\sigma_{n}\left(\ell_{n}\right)=\gamma\left(t_{n}\right)$ for each $n$ and $\sigma_{n} \rightarrow \sigma$ as $n \rightarrow \infty$. An asymptotic ray emanating from a fixed point is not unique in general, and the asymptotic relation is not symmetric, which means $\gamma$ may not be asymptotic to $\sigma$.

In a Hadamard manifold, this notion of asymptotic coincides with the old one, and in particular it is an equivalence relation and makes it possible to define the ideal boundary. If one wants to define the ideal boundary by an equivalence relation of rays, the asymptotic relation would be the first candidate. However, our asymptotic relation is not symmetric, and therefore other relations have been used (e.g., [7], [11]). For a manifold without conjugate points, this problem has been studied for instance in [5]. We will show that for a surface admitting total curvature the symmetry of the asymptotic relation has a rather strong implication (see [Lemma 2.6]), and give an explicit example of a surface where the relation is not symmetric. 
Even though our discussion in the next section can be applied to any open surface admitting total curvature, a special case of interest is a surface with nonnegative curvature. In fact, all the examples in the last section have nonnegative curvature. Every open surface with nonnegative curvature admits total curvature, and by Cohn-Vossen's result it is either simply connected or flat. Hence if $M$ is not contactible, then it is either a flat cylinder or a Möbius strip. From now on, we will assume that our surface $M$ is diffeomorphic to $\mathbb{R}^{2}$. In the case of nonnegative curvature we do not miss many, and in general this will make our arguments simple.

\section{CONVEXITY OF THE IDEAL BOUNDARY}

Let $M$ be a simply connected complete open surface admitting total curvature. In this section we will introduce the notion of convexity of the ideal boundary. Strictly convex, convex, and weakly convex ideal boundary will be defined, and in the next section we will give examples separating these definitions. A Hadamard manifold will clearly satisfy all the necessary conditions for the convexity we will define, and hence we may say our notion of convexity in fact describes how much an open surface fails to be a Hadamard manifold.

Let $D \subset M$ be a simply connected domain with piecewise smooth boundary $\partial D$, oriented relatively to $D$; and denote by $\kappa(\partial D)$ the sum of the geodesic curvature integral of $\partial D$ and of the exterior angles where $\partial D$ is not smooth. If $D$ is bounded, then by the Gauss-Bonnet theorem we have

$$
\kappa(\partial D)=2 \pi-\int_{D} K_{M}
$$

For this reason, we interpret the number in the right-hand side as the total convexity of the boundary $\partial D$. If $D$ is not bounded and $\partial D$ consists of a curve $c:(-\infty, \infty) \rightarrow M$ such that $c((-\infty, \alpha])$ and $c([\beta,+\infty))$ are rays for some $\alpha \leq \beta$, then by Cohn-Vossen [4] we have

$$
\kappa(\partial D) \leq \pi-\int_{D} K_{M}
$$

For a point $p \in M$ let $\sigma, \gamma:[0, \infty) \rightarrow M$ be two rays such that $\sigma(0)=$ $\gamma(0)=p$. Unless $\sigma \equiv \gamma$, the union of these two rays separates $M$ into two connected domains, which we will call ideal triangles since each of them looks like an infinitely large triangle with one edge contained in the ideal boundary. If $\Delta$ is such an ideal triangle with an interior angle $\theta=\varangle\left(\sigma^{\prime}(0), \gamma^{\prime}(0)\right)$, then we have

$$
\pi-\theta=\kappa(\partial \Delta) \leq \pi-\int_{\Delta} K_{M}
$$

and hence $\theta-\int_{\Delta} K_{M} \geq 0$. If the inequality is strict, we call the positive number, $\theta-\int_{\Delta} K_{M}$, the shortage of total curvature, and interpret it as the total convexity of the edge contained in the ideal boundary. This observation leads us to the following definition.

Definition 2.1. A surface $M$ is said to have strictly convex boundary at infinity if for any ideal triangle $\Delta$ the equality $\int_{\Delta} K_{M}=\theta$ implies that the Busemannfunctions $b_{\sigma}$ and $b_{\gamma}$ are the same.

For any $p \in M$ and a pair of rays emanating from $p$, we have two ideal triangles. If $\partial M$ is strictly convex and either one of these two ideal triangles has 
no shortage of total curvature, then these two rays define the same Busemannfunction. If we assume that a ray intersects the ideal boundary perpendicularly (if anyone wants to consider such an angle), our definition of strictly convex boundary is a direct generalization of a compact domain with strictly convex boundary. In fact, what we are trying to do with the ideal boundary is an imitation of the convexity of a compact domain.

Let $M$ be an open manifold and $C l(M) \subset C_{*}(M)$ be the compactification of $M$ by horofunctions. Maybe the most significant difference between $C l(M)$ and a compact domain with boundary is the following. Every boundary point of a compact domain can be connected to an interior point by a minimal geodesic. Suppose a horofunction $h \in \partial M$ is connected to an interior point $p \in M$ by a ray $\gamma:[0, \infty) \rightarrow M$. Then $h$ is the limit of the equivalence class $\bar{d}_{\gamma(t)}$. Therefore, in fact, we are looking for a ray defining a Busemann-function which agrees with $h$ up to a constant. It is not always possible to find such a ray in general, and we need the following notion of convexity.

Definition 2.2. A surface $M$ is said to have convex boundary at infinity if for any $p \in M$ and any horofunction $h \in \partial M$ there is a ray $\gamma:[0, \infty) \rightarrow M$ such that $\gamma(0)=p$ and the Busemann-function $b_{\gamma}$ agrees with $h$ up to a constant.

In a sense, we may say this definition is a generalization of a bounded convex domain. Since $M$ is complete, any two interior points can be connected by a minimal geodesic by the Hopf-Rinow theorem. A ray plays the role of a minimal connection between an interior point and a boundary point at infinity. A minimal connection between two boundary points can be obtained as a limit of minimal geodesics as their end points diverge to infinity. If this sequence of geodesics converges to a geodesic in $M$, then the limit is called a line. If a surface $M$ diffeomorphic to $\mathbb{R}^{2}$ admits total curvature and there exists a line on $M$, then we have [4]

$$
\int_{M} K_{M} \leq 0
$$

For instance, if we assume that the curvature is nonnegative, then $M$ must be flat. We can obtain the same conclusion from the Toponogov splitting theorem as well. If $M$ is a visibility manifold, then by definition every boundary points are connected by lines in $M$. In general, however, a sequence of geodesics will diverge to infinity as their end points do.

In [1], it was shown that for a Hadamard manifold every horofunction is the Busemann-function, and hence the ideal boundary can be defined by the equivalence classes of rays emanating from any fixed point of $M$. The following theorem is a generalization of this fact for surfaces admitting total curvature. One of our main objects is to prove this theorem, which will be stated again later.

Theorem. A surface $M$ with strictly convex boundary has convex boundary at infinity.

The definition of strictly convex boundary does not involve the notion of horofunctions, and it turns out they are not very easy to deal with. For this reason, we will first prove a weaker statement with the following weaker convexity. 
Definition 2.3. A surface $M$ is said to have weakly convex boundary if for any $p \in M$ and any Busemann-function $b_{\gamma}$ defined by a ray $\gamma$ there is a ray $\sigma:[0, \infty) \rightarrow M$ such that $\sigma(0)=p$ and $b_{\sigma}$ agrees with $b_{\gamma}$ up to a constant.

Since every Busemann-function is a horofunction, it is clear that convex boundary implies weakly convex boundary. The converse is not true, and we will have an example. If every horofunction is a Busemann-function for some ray, then the convexity and the weak convexity are obviously equivalent. Hence we may say that this is actually the difference between a compact domain and a compactified open manifold.

For any ray $\gamma$ the Busemann-function $b_{\gamma}: M \rightarrow \mathbb{R}$ is not differentiable in general, and the nondifferentiability can be studied by the asymptotic relation of rays. Denote by $\bar{\nabla} b_{\gamma}$ the closure in the tangent bundle $T M$ of the set of gradient vectors of $b_{\gamma}$ at all points of differentiability. At each $p \in M$ the set $\bar{\nabla} b_{\gamma}(p) \stackrel{\text { def }}{=} \bar{\nabla} b_{\gamma} \cap T_{p} M$ coincides with the set of unit vectors at $p$ tangent to rays asymptotic to $\gamma$ (see [9, Theorem 1.1]). A point $p \in M$ is called a regular point if $b_{\gamma}$ is differentiable there, and hence $\bar{\nabla} b_{\gamma}(p)$ is a single vector. Since a Busemann-function is a Lipschitz continuous function with the Lipschitz constant one, it is differentiable almost everywhere.

If a ray $\sigma:[0, \infty) \rightarrow M$ is asymptotic to a ray $\gamma$, then $b_{\gamma}$ is differentiable at $\sigma(t)$ for each $t>0$ and the tangent vector $\sigma^{\prime}(t)$ is the gradient vector of $b_{\gamma}$. Hence $b_{\gamma}-b_{\sigma}$ is a constant along the ray $\sigma$. The converse is also clearly true. We further have the following simple lemma for later use.

Lemma 2.4 (compare [9, Lemma 1.2]). If $\sigma:[0, \infty) \rightarrow M$ is a ray asymptotic to a ray $\gamma$, then for each $x \in M$

$$
b_{\gamma}(x) \leq b_{\gamma}(\sigma(0))+b_{\sigma}(x) .
$$

Proof. For any $x \in M$ and any $\varepsilon>0$, there exists a number $t>0$ such that $b_{\sigma}(x) \geq d(x, \sigma(t))-t-\varepsilon$ since $b_{\sigma}(x)=\lim _{t \rightarrow \infty} d(x, \sigma(t))-t$. By the triangle inequality,

$$
b_{\gamma}(x) \leq b_{\gamma}(\sigma(t))+d(x, \sigma(t)) .
$$

Since $b_{\gamma}-b_{\sigma}$ is a constant along $\sigma$, we have $b_{\gamma}(\sigma(t))=b_{\gamma}(\sigma(0))-t$, and hence

$$
b_{\gamma}(x) \leq b_{\gamma}(\sigma(0))+b_{\sigma}(x)+\varepsilon .
$$

If $\sigma$ and $\gamma$ are two rays asymptotic to each other, then $b_{\gamma}-b_{\sigma}$ is constant along $\sigma$ and $\gamma$. In general, however, the constant along $\sigma$ may not be the same as the constant along $\gamma$. We will give an example.

There is another small fact we must consider when we deal with the asymptotic relation of rays. Suppose $\sigma:[0, \infty) \rightarrow M$ is a ray and there is a number $t>0$ such that the restriction $\left.\sigma\right|_{[t, \infty}$ of $\sigma$ on the interval $[t, \infty)$ is asymptotic to a ray $\gamma$. Unfortunately this does not imply that $\sigma$ is asymptotic to $\gamma$ in general.

These problems may be related and deserve more study. But for the moment we only need the following corollary, which can be proved by the same argument as the lemma and we shall omit the proof.

Corollary 2.5. If $\sigma, \gamma:[0, \infty) \rightarrow M$ are two rays and there are constants $T>0$ and $a$ such that

$$
b_{\sigma}(\sigma(t))-b_{\gamma}(\sigma(t))=b_{\sigma}(\gamma(t))-b_{\gamma}(\gamma(t))=a
$$

for all $t \geq T$, then $b_{\sigma}-b_{\gamma} \equiv a$. 
When two rays are asymptotic to each other and the Busemann-functions agree along the rays, the corollary implies that they are in fact identical. This fact is used in the following lemma.

Lemma 2.6. If the asymptotic relation is symmetric, then for any ray $\sigma$ asymptotic to a ray $\gamma$ the Busemann-functions $b_{\gamma}$ and $b_{\sigma}$ agree up to a constant.

Proof. Let $\sigma$ be a ray asymptotic to a ray $\gamma$. Then of course $\gamma$ is also asymptotic to $\sigma$ by the assumption. Since $M$ is simply connected, there exists a compact set $C \subset M$ such that $M-(C \cup \sigma \cup \gamma)$ has two connected components, each of which is homeomorphic to a strip with one open end. Since $\sigma$ is asymptotic to $\gamma$, one of these two domains contains a sequence $\left\{\sigma_{n}\right\}$ of minimal geodesics such that $\sigma_{n} \rightarrow \sigma$ as $n \rightarrow \infty$. We denote this domain by $\Sigma$ and find a number $t_{0}$ such that $b_{\gamma}(p) \geq-t_{0}$ for each $p \in C$.

For any interior point $x \in \Sigma$ with $b_{y}(x) \leq-t_{0}$, let $\gamma_{x}$ be a ray asymptotic to $\gamma$ and emanating from $x$. We first claim that $\gamma_{x}$ is contained in $\Sigma$. If $\gamma_{x}$ intersects either $\sigma$ or $\gamma$, the intersection point cannot be a regular point of $b_{\gamma}$ because $\bar{\nabla} b_{\gamma}$ is not a single vector there. Since every interior point of a ray is a regular point, the intersection point, if any, is not an interior point of $\gamma$ or $\sigma$. Moreover, $\gamma_{x}$ cannot intersect $C$ because $b_{\gamma} \circ \gamma_{x}$ is a decreasing function. Therefore $\gamma_{x}$ does not intersect the boundary of $\Sigma$, and hence it must be contained in $\Sigma$.

We next claim that there is a subsequence $\left\{\sigma_{k}\right\}$ of $\left\{\sigma_{n}\right\}$ such that $\sigma_{k}$ intersect $\gamma_{x}$ at $\gamma_{x}\left(t_{k}\right)$ and $t_{k} \rightarrow \infty$ as $k \rightarrow \infty$. Then it follows that $\sigma$ is aymptotic to $\gamma_{x}$. If our claim is not true, then there is a number $T>0$ such that $\gamma_{x}(T)$ is not contained in the triangle determined by $\gamma, \sigma_{n}$, and the boundary of $C$ for each $n$. Then after taking the limit of $\sigma_{n}$ we see that for $t>T \gamma_{x}(t)$ is not contained in $\Sigma$, which is a contradiction.

By assumption, we know $\gamma_{x}$ is asymptotic to both $\sigma$ and $\gamma$, and the Busemann-functions $b_{\sigma}$ and $b_{\gamma}$ agree up to a constant along $\gamma_{x}$. Therefore, $b_{\sigma}$ and $b_{\gamma}$ have the same gradient vector whenever they are both differentiable, and hence $\bar{\nabla} b_{\sigma}=\bar{\nabla} b_{\gamma}$. Then it is easy to see that $b_{\sigma}-b_{\gamma}$ is a constant on $\Sigma$ except some compact set and by Corollary $2.5, b_{\sigma}$ and $b_{\gamma}$ agree on $M$ up to a constant.

Corollary 2.7. A surface $M$ has weakly convex boundary if and only if the asymptotic relation is symmetric.

Proof. Suppose the asymptotic relation is symmetric. For any Busemannfunction $b_{\gamma}$ defined by a ray $\gamma$, we obtain a ray $\sigma$ emanating from $p$ and asymptotic to $\gamma$ for each $p \in M$. By the lemma, the Busemann-functions $b_{\gamma}$ and $b_{\sigma}$ agree up to a constant, and therefore $M$ has weakly convex boundary.

We now assume that $\partial M$ is weakly convex. Let $\sigma:[0, \infty) \rightarrow M$ be a ray asymptotic to a ray $\gamma$. For any $\varepsilon>0$ there must be a ray emanating from $\sigma(\varepsilon)$ and defining the same Busemann-function as $b_{\gamma}$ up to a constant. But $\sigma(\varepsilon)$ is a regular point of $b_{\gamma}$, and hence $\left.\sigma\right|_{[\varepsilon, \infty)}$ is the only candidate. Therefore $b_{\gamma}$ and $b_{\sigma}$ agree up to a constant, and $\gamma$ is asymptotic to $\sigma$.

In the definition of strictly convex ideal boundary, we only considered two rays emanating from the same point. But for convex or weakly convex boundary we have to compare rays with different origins. Let $\gamma:[0, \infty) \rightarrow M$ be a ray 
and $p \in M$ a point away from $\gamma[0, \infty)$. For a monotone divergent sequence $\left\{t_{n}\right\}$ and minimal geodesics $\left\{\sigma_{n}\right\}$ from $p$ to $\gamma\left(t_{n}\right)$ we consider the geodesic triangles determined by $\sigma_{n}, \gamma\left[0, t_{n}\right]$, and the minimal geodesic $c$ from $\gamma(0)$ to $p$. Denote this triangle by $T_{n}$ for each $n$. It is clear that for some subsequence $\left\{\sigma_{k}\right\}$ of $\left\{\sigma_{n}\right\}$ the sequence $\left\{T_{k}\right\}$ is monotone increasing, and $\Sigma \stackrel{\text { def }}{=} \bigcup_{k=1}^{\infty} T_{k}$ is a domain homeomorphic to a strip with one open end and $\partial \Sigma$ consists of $c$, $\gamma$, and a ray $\sigma$ asymptotic to $\gamma$. Then by Cohn-Vossen we have

$$
(\pi-\theta)+(\pi-\varphi)=\kappa(\partial \Sigma) \leq \pi-\int_{\Sigma} K_{M},
$$

where $\theta$ and $\varphi$ are two interior angles of $\Sigma$. On the other hand, for each $T_{k}$, we have $\kappa\left(\partial T_{k}\right)=2 \pi-\int_{T_{k}} K_{M}$ by the Gauss-Bonnet formula. After taking $k \rightarrow \infty$, we easily see that

$$
\int_{\Sigma} K_{M}=\theta+\varphi-\pi
$$

With the help of this result, we can now compare two rays with different origins in a surface with strictly convex boundary.

Lemma 2.8. Let $M$ be a surface with strictly convex ideal boundary. For any $\Sigma$ determined by two rays and a minimal geodesic with the interior angles $\theta$ and $\varphi$, the equality $\int_{\Sigma} K_{M}=\theta+\varphi-\pi$ implies that the rays are asymptotic to each other.

Proof. Let $\sigma, \gamma:[0, \infty) \rightarrow M$ be two rays, and let $c[0, \ell] \rightarrow M$ be a minimal geodesic from $\gamma(0)$ to $\sigma(0)$. Let $\Sigma$ be the strip enclosed by $\sigma, \gamma$, and $c$ with the interior angles $\varphi$ at $\gamma(0)=c(0)$ and $\theta$ at $\sigma(0)=c(\ell)$. If $\left\{t_{n}\right\}$ and $\left\{\sigma_{n}\right\}$ are as usual, a limit $\bar{\sigma}$ of $\left\{\sigma_{n}\right\}$ is a ray emanating from $\sigma(0)$ and asymptotic to $\gamma$. Assume that $\bar{\sigma}$ is different from $\sigma$. Then either a subsequence of $\left\{\sigma_{n}\right\}$ is contained in $\Sigma$ and hence $\bar{\sigma}$ is contained in $\Sigma$ or a subsequence of $\left\{\sigma_{n}\right\}$ is contained in the closure of $M-\Sigma$ and hence $\bar{\sigma}$ does not intersect $\Sigma$ except at $\sigma(0)=\bar{\sigma}(0)$.

In either case, we obtain a strip $\Sigma^{\prime}$ enclosed by $\gamma, \bar{\sigma}$, and $c$ with the interior angles $\varphi^{\prime}$ at $\gamma(0)$ and $\theta^{\prime}$ at $\sigma(0)$. Then clearly

$$
\int_{\Sigma^{\prime}} K_{M}=\theta^{\prime}+\varphi^{\prime}-\pi
$$

In the first case, $\Sigma^{\prime}$ is contained in $\Sigma$ and $\varphi^{\prime}=\varphi, \theta^{\prime}<\theta$. Then for the ideal triangle $\Delta$ determined by the difference of $\Sigma$ and $\Sigma^{\prime}$, we have

$$
\int_{\Delta} K_{M}=\int_{\Sigma} K_{M}-\int_{\Sigma^{\prime}} K_{M}=\theta-\theta^{\prime},
$$

where $\theta-\theta^{\prime}$ is the interior angle of $\Delta$. Therefore, by the assumption, $\sigma$ and $\bar{\sigma}$ define the same Busemann-function. In the second case, $\Sigma$ and $\Sigma^{\prime}$ intersect only along the boundary and $\varphi+\varphi^{\prime}=2 \pi$. Then for the ideal triangle $\Delta$ determined by the union of $\Sigma$ and $\Sigma^{\prime}$, we have

$$
\int_{\Delta} K_{M}=\int_{\Sigma} K_{M}+\int_{\Sigma^{\prime}} K_{M}=\theta+\theta^{\prime},
$$


where $\theta+\theta^{\prime}$ is the interior angle of $\Delta$, and we obtain the same conclusion.

Since $\bar{\sigma}$ is asymptotic to $\gamma$, by Lemma 2.4 for each $t \geq 0$ we have

$$
b_{\gamma}(\sigma(t)) \leq b_{\gamma}(\bar{\sigma}(0))+b_{\sigma}(\sigma(t)) .
$$

However, $\bar{\sigma}(0)=\sigma(0)$ and $b_{\delta}(\sigma(t))=b_{\sigma}(\sigma(t))=-t$, and hence $b_{\gamma}(\sigma(t)) \leq$ $b_{\gamma}(\sigma(0))-t$ for each $t \geq 0$. On the other hand, $b_{\gamma}$ is a Lipschitz function, and $b_{\gamma}(\sigma(0))-b_{\gamma}(\sigma(t)) \leq t$. Therefore $b_{\sigma}-b_{\gamma}$ is a constant along $\sigma$, and hence $\sigma$ is asymptotic to $\gamma$. Similarly, $\gamma$ is asymptotic to $\sigma$.

Theorem 2.9. A surface with strictly convex boundary has weakly convex boundary at infinity.

Proof. For any ray $\gamma$ and a point $p \in M$ let $\sigma$ be a ray emanating from $p$ and asymptotic to $\gamma$. We will show that $\gamma$ is also asymptotic to $\sigma$. Then the theorem follows by Corollary 2.7.

If $p$ is an interior point of $\gamma$, then clearly $\sigma$ is contained in $\gamma[0, \infty)$. And if $p=\gamma(0)$, we may consider $\left.\sigma\right|_{[\varepsilon, \infty)}$ for some $\varepsilon>0$. Hence, without loss of generality, we assume $p$ is a point away from $\gamma$. Then there is a strip $\Sigma$ enclosed by $\sigma, \gamma$, and a minimal geodesic from $\gamma(0)$ to $\sigma(0)$ so that there is no shortage of the total curvature for $\Sigma$. By Lemma 2.8, $\gamma$ is asymptotic to $\sigma$.

We now consider $C l(M)$, the closure of $M$ in $C_{*}(M)$. A point $x \in M$ is identified with the equivalence class $\bar{d}_{x}$ of the distance function $d_{x}$, and a point in $\partial M$ is the equivalence class of horofunctions. For any $\bar{h} \in \partial M$, there is a horofunction $h \in C(M)$ such that $h(p)=0$ for a fixed point $p \in M$. Then $h$ is a Lipschitz function with the Lipschitz constant one, and there is a divergent sequence $\left\{x_{n}\right\}$ such that the equivalence class $\bar{d}_{x_{n}}$ can be represented by $f_{n} \in C(M)$ with $f_{n}(p)=0$ for each $n$ and the sequence $\left\{f_{n}\right\}$ uniformly converges to $h$ on compact sets.

We call a ray $\sigma$ asymptotic to $h$ if there exists a sequence $\left\{x_{k}\right\}$ and a sequence $\left\{\sigma_{k}:\left[0, \ell_{k}\right] \rightarrow M\right\}$ of minimal geodesics such that $\sigma_{k}\left(\ell_{k}\right)=x_{k}$ for each $k$, and as $k \rightarrow \infty \quad \sigma_{k} \rightarrow \sigma$ and $\bar{d}_{x_{k}} \rightarrow \bar{h}$ in $C_{*}(M)$.

For any interval $[0, \tau]$ we can choose $k$ sufficiently large so that the distance function $d_{x_{k}}$ is arbitrarily close to $h+\alpha$ on $\sigma[0, \tau]$ for a constant $\alpha$ and $\sigma_{k}[0, \tau]$ is arbitrarily close to $\sigma[0, \tau]$. Therefore, $h-b_{\sigma}$ is a constant along $\sigma$, and hence the velocity vector of $\sigma$ is the gradient vector of $h$ with the opposite direction. By the same argument as the Busemann-function, we see that $\bar{\nabla} h(p)$ is the set of unit vectors at $p$ tangent to rays asymptotic to $h$, and $\sigma$ is asymptotic to $h$ if and only if $h-b_{\sigma}$ is a constant along $\sigma$. Regular points of $h$ are defined in the same fashion, and any interior point of an asymptotic ray is a regular point of $h$.

We are now ready to prove

Theorem 2.10. A surface $M$ with strictly convex boundary has convex boundary. Proof. By Theorem 2.9 it suffices to show that every horofunction is a Busemann-function defined by some ray.

Let $h$ be a horofunction and $p \in M$ a regular point of $h$. Assume that $h(p)=0$ and $\gamma$ is a ray asymptotic to $h$ and emanating from $p$. We claim that the Busemann-function $b_{\gamma}$ is the same as $h$. 
For any $q \in M$, which is a regular point of $h$, let $\sigma$ be a ray asymptotic to $h$ and emanating from $q$. If $h$ is defined by a divergent sequence $\left\{x_{n}\right\}$, then the sequence of minimal connections between $p$ and $x_{n}$ has a convergent subsequence and the limit must coincide with $\gamma$ since $p$ is a regular point of $h$. After taking a subsequence of this subsequence if necessary, we may assume that $\gamma$ and $\sigma$ are limits of minimal geodesics $\left\{\gamma_{k}:\left[0, \ell_{k}\right] \rightarrow M\right\}$ and $\left\{\sigma_{k}:\left[0, m_{k}\right] \rightarrow M\right\}$ such that $\gamma_{k}(0)=p, \sigma_{k}(0)=q$, and $\gamma_{k}\left(\ell_{k}\right)=\sigma_{k}\left(m_{k}\right)=x_{k}$ for each $k$.

Let $c:[0, \ell] \rightarrow M$ be a minimal geodesic such that $c(0)=p, c(\ell)=q$. Then $\gamma \cup \sigma \cup c$ divides $M$ into two connected domains, each of which is diffeomorphic to a strip with one open end. Since the minimal geodesics $\left\{\gamma_{k}\right\}$, $\left\{\sigma_{k}\right\}$ do not intersect $c$, we have two possibilities. The first is that there is a subsequence $\left\{\gamma_{i}\right\}$ (or $\left\{\sigma_{i}\right\}$ ) of $\left\{\gamma_{k}\right\}$ (resp., $\left\{\sigma_{k}\right\}$ ) such that $\gamma_{i}$ (resp., $\sigma_{i}$ ) intersects $\sigma$ (resp., $\gamma$ ) at $\sigma\left(t_{i}\right)$ (resp., $\gamma\left(t_{i}\right)$ ) for each $i$. Then the sequence $\left\{t_{i}\right\}$ has no accumulation point because it would force the two rays to intersect. Since we already know that the asymptotic relation is symmetric, we can conclude in this case that $\gamma$ and $\sigma$ are asymptotic to each other, and hence define the same Busemann-function up to a constant.

The second case is when there is a subsequence $\left\{x_{i}\right\}$ of $\left\{x_{k}\right\}$ such that $\sigma\left(\right.$ and $\gamma_{i}$ ) does not intersect $\gamma$ (resp., $\sigma_{i}$ ) for each $i$. In this case, after taking a subsequence if necessary, we may assume that the geodesic triangles determined by $\sigma_{i}, \gamma_{i}$, and $c$ are all contained in one of two strips, which we denote by $\Sigma$. Denote by $T_{i}$ the triangle for each $i$. After taking a subsequence once more if necessary, we assume $\left\{T_{i}\right\}$ is monotone increasing sequence such that $\Sigma=\bigcup_{i=1}^{\infty} T_{i}$. Then by the Gauss-Bonnet formula for each $T_{i}$ and CohnVossen's result for $\Sigma$, we see that there cannot be any shortage of the total curvature for $\Sigma$, and hence $\sigma$ and $\gamma$ define the same Busemann-function up to a constant by Lemma 2.8 .

Now together with the fact that $h-b_{\sigma}$ is a constant along $\sigma$, we conclude that $h$ and $b_{\gamma}$ have the same gradient vector whenever $h$ is differentiable. Therefore $\bar{\nabla} h=\bar{\nabla} b_{\gamma}$, and hence $h-b_{\gamma}$ is a constant, which is a zero since $h(p)=b_{\gamma}(p)=0$.

\section{EXAMPLES}

In the previous section, we defined three kinds of convexity for the ideal boundary. In this section we will produce examples separating them. All the examples we will present in this section have nonnegative curvature, and hence they all admit nonnegative total curvature bounded by $2 \pi$.

Before we produce more complicated examples, we can easily see there is a trivial example of a surface with strictly convex boundary which is not Hadamard. Consider the paraboloid $z=x^{2}+y^{2}$ in $\mathbb{R}^{3}$. It is easy to check that the rays are all asymptotic to each other and they define the same Busemannfunction. Therefore there is only one point at infinity, and hence the paraboloid has strictly convex boundary at infinity.

The first kind of nontrivial examples we will consider are surfaces of revolution in $\mathbb{R}^{3}$. Let $M$ be a surface of revolution with the parametrization

$$
x=f(u) \cos v, \quad y=f(u) \sin v, \quad z=u,
$$


where $f$ is defined for $u \geq 0$ and $f(0)=0$. Then $M$ is a surface in the upper half space, and the origin is the vertex which, we always assume, has a smooth neighborhood. We further assume $f^{\prime}$ is a nonnegative nonincreasing function of $u$ for $M$ to be an open nonnegatively curved manifold. Then by looking at the Gauss map,

$$
\int_{M} K_{M}=2 \pi\left(1-\frac{\alpha}{\sqrt{1+\alpha^{2}}}\right),
$$

where $\alpha=\lim _{u \rightarrow \infty} f^{\prime}(u)$.

Let $\gamma:\left[t_{0}, \infty\right) \rightarrow M$ be a geodesic with the parametrization

$$
u=t, \quad v=g(t) .
$$

Of course, $\gamma$ does not have constant speed (hence strictly speaking is not a geodesic), but we are only interested in its image in $M$. Let $\theta(t)$ denote the angle between the tangent vector of $\gamma$ and the parallel $z=t$ for each $t \in\left[t_{0}, \infty\right)$. Then by Clairaut's relation we have

$$
f(t) \cos \theta(t)=\text { const }=|c|
$$

and

$$
g^{\prime}(t)=\frac{c}{f}\left(\frac{f^{\prime 2}+1}{f^{2}-c^{2}}\right)^{1 / 2},
$$

where the sign of $c$ is determined by the direction of $\gamma$ winding around $M$.

Suppose $\gamma:\left[t_{0}, \infty\right) \rightarrow M$ is a geodesic with $c>0$ and

$$
\int_{t_{0}}^{\infty} g^{\prime}(t) d t \stackrel{\text { def }}{=} \Delta g \leq \pi
$$

Then any other geodesic with $|\Delta g| \leq \pi$ emanating from $\gamma\left(t_{0}\right)$ does not intersect $\gamma$, and hence $\gamma$ is a ray. Furthermore, it is easy to see that $\Delta g$ is an increasing function of $c>0$ for geodesics emanating from $\gamma\left(t_{0}\right)$, and $\gamma$ is a ray asymptotic to the meridian $v=\lim _{t \rightarrow \infty} g(t)=g\left(t_{0}\right)+\Delta g$. In fact, any two rays $\gamma_{1}, \gamma_{2}$ determined by $g_{1}(t), g_{2}(t)$ respectively with different origins are asymptotic to each other if and only if

$$
\lim _{t \rightarrow \infty} g_{1}(t)=\lim _{t \rightarrow \infty} g_{2}(t) .
$$

In particular, the asymptotic relation is symmetric on a surface of revolution.

With this observation, we can prove

Proposition 3.1. A surface of revolution has convex boundary at infinity.

Proof. Let $h$ be a horofunction defined by a sequence $\left\{x_{n}\right\}$ such that $h\left(q_{0}\right)=0$ for a fixed point $q_{0} \in M$ other than the vertex, and let $c:[0, \ell] \rightarrow M$ be the parallel containing $q_{0}$. Then the compact domain bounded by $c$ is convex, and we can find a sequence $\left\{p_{n}\right\}$ of points on $c[0, \ell]$ such that for each $n$

$$
d\left(x_{n}, p_{n}\right)=\inf \left\{d\left(x_{n}, q\right) \mid q \in c[0, \ell]\right\} .
$$

Since $c[0, \ell]$ is compact, we have an accumulation point $p \in c[0, \ell]$ of $\left\{p_{n}\right\}$. Then, by reducing to a subsequence, the meridian $\gamma$ through $p$ is the limit of minimal geodesics connecting $p_{n}$ and $x_{n}$. Therefore $\gamma$ is a ray asymptotic $h$, and we claim the Busemann-function $b_{\gamma}$ is same as $h$. 
For any $y \in M$ consider a sequence $\left\{\sigma_{n}\right\}$ of minimal geodesics from $y$ to $x_{n}$ for each $n$. After taking a subsequence if necessary, we may assume that either all of $\left\{\sigma_{n}\right\}$ intersect $\gamma$, or none of them intersects $\gamma$. In the first case, the intersection points on $\gamma$ must diverge, and hence the limit ray $\sigma$ is asymptotic to $\gamma$.

In the second case, we assume that $\sigma$ is not asymptotic to $\gamma$ and obtain a contradiction. Suppose $\sigma$ and $\sigma_{n}$ for each $n$ are parameterized by $g$ and $g_{n}$ respectively. By assumption, $\lim _{t \rightarrow \infty} g(t)$ is not the same as the $v$-coordinate of the point $p$. For $n$ sufficiently large, the initial condition of $\sigma_{n}$ is close to that of $\sigma$ and $\left|\Delta g_{n}\right|<|\Delta g|+\varepsilon$ for arbitrarily small $\varepsilon>0$. Then $p_{n}$ is not close to $p$, which is a contradiction. Therefore any ray asymptotic to $h$ is asymptotic to $\gamma$, and hence $h=b_{\gamma}$.

Example 3.2. A surface with ideal boundary which is convex but not strictly convex.

Let $M$ be the surface of revolution defined by the function $f(u)=u^{3 / 4}$. We assume that the neighborhood of the vertex is smooth. Since $\lim _{u \rightarrow \infty} f^{\prime}(u)=0$, we have

$$
\int_{M} K_{M}=2 \pi
$$

and hence there is no shortage of total curvature for any ideal triangle determined by two meridians. However for sufficiently large $t$,

$$
g^{\prime}(t)=\frac{c}{f}\left(\frac{f^{\prime 2}+1}{f^{2}-c^{2}}\right)^{1 / 2}=O\left(t^{-\frac{3}{2}}\right),
$$

which has a bounded integral. Hence we can find a ray which is not a meridian but asymptotic to some meridian. Therefore, two meridians are not asymptotic to each other, and hence $\partial M$ is not strictly convex. However by Proposition 3.1 $\partial M$ is convex.

We now consider the second class of examples, which are surfaces embedded in $\mathbb{R}^{3}$.

Let $f: \mathbb{R} \rightarrow \mathbb{R}$ be a smooth function such that $f(0)=0, f(-x)=f(x)$, $f(x) \geq 0, f^{\prime \prime}(x) \geq 0$, and $f^{\prime}(x) \rightarrow \infty$ as $|x| \rightarrow \infty$. Then clearly $f(x) \rightarrow \infty$ as $|x| \rightarrow \infty$. In $\mathbb{R}^{3}$, define two flat surfaces,

$$
\begin{aligned}
& R_{1}=\{(x, y, 0) \mid y \geq f(x)\}, \\
& R_{2}=\{(x, y, \alpha) \mid y \geq f(x)\},
\end{aligned}
$$

where $\alpha$ is a small constant. Along the boundary we connect $R_{1}$ and $R_{2}$ with an infinitely long patch to make it a smooth surface diffeomorphic to $\mathbb{R}^{2}$. In fact, we obtain an open surface $M$ in $\mathbb{R}^{3}$ which looks like a large flat envelope with round edge.

Example 3.3. A surface with ideal boundary which is weakly convex but not convex.

Let $M$ be as above with $f(x)=|x|^{\frac{3}{2}}$. This function is not smooth at the origin, but we can take care of that problem when we put a narrow patch along the boundary. For each $x \in \mathbb{R}$ let $p_{x} \in R_{1}, q_{x} \in R_{2}$ be two points with 
coordinates $(x, f(x), 0)$ and $(x, f(x), \alpha)$ respectively. We make the patch so that for any $p \in R_{1}$ we have

$$
d\left(p, p_{x}\right) \leq d\left(p, q_{x}\right) \leq d\left(p, p_{x}\right)+\delta
$$

for a fixed constant $\delta>0$, and similarly for $q \in R_{2}$.

In each flat region, the only rays are the vertical (parallel to the $y$-axis) lines. We will show that two rays one from each side are not asymptotic. Since all the verticall lines in each flat area define the same Busemann-function up to constants, we can look at any ray.

Let $\gamma$ be a vertical line in $R_{2}$. For any $p \in R_{1}$ let $\left\{t_{n}\right\}$ be a monotone divergent sequence and let $\left\{\sigma_{n}\right\}$ be minimal geodesics from $p$ to $\gamma\left(t_{n}\right)$ for each $n$. For each $n$ let $p_{n} \in \partial R_{1}$ be the point where $\sigma_{n}$ intersects the curve $y=f(x)$. Without loss of generality we assume the coordinates of $p_{n}$ are $\left(x_{n}, x_{n}^{3 / 2}, 0\right)$ for each $n$. If $\left\{\sigma_{n}\right\}$ converges to a vertical line in $R_{1}$, we must have $x_{n} \rightarrow \infty$ as $n \rightarrow \infty$.

For each $n$ let $q_{n}=\left(x_{n}, x_{n}^{3 / 2}, \alpha\right) \in \partial R_{2}$ be the point corresponding to $p_{n}$. Then by the triangle inequality, we have

$$
b_{\gamma}(p) \leq b_{\gamma}\left(q_{1}\right)+d\left(p, q_{1}\right) .
$$

Since $\left\{\sigma_{n}\right\}$ converges to $\sigma$, for any $\varepsilon>0$ there exists a number $N$ such that for each $n \geq N$

$$
\begin{aligned}
b_{\gamma}(p) & \geq d\left(p, \gamma\left(t_{n}\right)\right)-t_{n}-\varepsilon \\
& \geq d\left(p, q_{n}\right)+b_{\gamma}\left(q_{n}\right)-\varepsilon-\delta \\
& \geq d\left(p, p_{n}\right)+b_{\gamma}\left(q_{n}\right)-\varepsilon-\delta .
\end{aligned}
$$

Combining two inequalities with $b_{\gamma}\left(q_{1}\right)-b_{\gamma}\left(q_{n}\right)=x_{n}^{3 / 2}-x_{1}^{3 / 2}$, we have

$$
d\left(p, p_{n}\right)-x_{n}^{3 / 2} \leq d\left(p, q_{1}\right)-x_{1}^{3 / 2}+\varepsilon+\delta .
$$

However, if $p=(a, b, 0)$, the left-hand side is

$$
\sqrt{\left(x_{n}-a\right)^{2}+\left(x_{n}^{3 / 2}-b\right)^{2}}-x_{n}^{3 / 2},
$$

which goes to infinity as $x_{n} \rightarrow \infty$, and it is a contradiction.

Since the width of the patch is bounded, it is easy to see that a ray cannot stay in the curved region. Therefore, if a ray is asymptotic to another ray, eventually they become vertical lines in one flat area, where they clearly define the same Busemann-function up to a constant. Then by Corollary 2.5 we conclude that $M$ is weakly convex.

To show $M$ is not convex, let $h$ be a horofunction defined by a divergent sequence $\left\{p_{n}\right\}$ of points in the curved area. In each flat area, a ray asymptotic to $h$ is a vertical line. If $\sigma \subset R_{1}, \gamma \subset R_{2}$ are rays emanating from the same horizontal level, then we have

$$
h(p)=\min \left\{b_{\sigma}(p), b_{\gamma}(p)\right\}+a
$$

for some constant $a$. Since $\sigma$ and $\gamma$ are not asymptotic, $b_{\sigma}$ and $b_{\gamma}$ are not in the same equivalence class and hence $h$ cannot be expressed as the Busemannfunction of a single ray.

Example 3.4. A surface with ideal boundary which is not weakly convex. 
Let $M$ be a surface defined in the same fashion as Example 3.3 with $f(x)=$ $\frac{1}{4} x^{2}$ and $\delta \leq \frac{1}{2}$. For each $x \in \mathbb{R}$ let $p_{x} \in \partial R_{1}, q_{x} \in \partial R_{2}$ as before. If $\gamma$ is a vertical line in $R_{2}$, we assume that

$$
b_{\gamma}\left(q_{x}\right)<b_{\gamma}\left(p_{x}\right)
$$

for each $x \in \mathbb{R}$, and $\lim _{|x| \rightarrow \infty}\left(b_{y}\left(p_{x}\right)-b_{y}\left(q_{x}\right)\right)=0$.

We see this can be done, for example, if we make the patch so that the minimal geodesic between $p_{x}$ and $q_{x}$ is perpendicular to the curve $y=f(x)$, or we obtain the patch by a parallel translation of the curve $y=f(x)$ along a short curve parallel to the $y z$-plane. Then the above inequality is obvious. And for sufficiently large $x$, the angle between the minimal geodesic and the vertical line from $q_{x}$ is close to a right angle, and hence by the Toponogov theorem the difference must be close to zero.

Suppose $\gamma$ is the vertical line emanating from $q=(1,1, \alpha) \in R_{2}$, and consider a ray $\sigma$ emanating from $p=(0,1,0) \in R_{1}$ and asymptotic to $\gamma$. If $\sigma$ is not a vertical line in $R_{1}$, it must intersect the curve $y=f(x)$, boundary of $R_{1}$, at $\left(x_{0}, f\left(x_{0}\right)\right)$ for some $x_{0}>0$. Put $p_{0}=\left(x_{0}, f\left(x_{0}\right), 0\right), q_{0}=$ $\left(x_{0}, f\left(x_{0}\right), \alpha\right)$. Then there is a number $\varepsilon>0$ such that

$$
\begin{aligned}
b_{\gamma}(p) & =b_{\gamma}\left(p_{0}\right)+d\left(p, p_{0}\right) \\
& =b_{\gamma}\left(q_{0}\right)+d\left(p, p_{0}\right)+\varepsilon .
\end{aligned}
$$

For any $x>0$, by the triangle inequality

$$
b_{\gamma}(p) \leq b_{\gamma}\left(p_{x}\right)+d\left(p, p_{x}\right) .
$$

However, for any $x>0$,

$$
\begin{aligned}
d\left(p, p_{x}\right) & =\sqrt{x^{2}+\left(\frac{1}{4} x^{2}-1\right)^{2}}=\frac{1}{4} x^{2}+1, \\
b_{y}\left(q_{x}\right) & =1-\frac{1}{4} x^{2},
\end{aligned}
$$

and hence $b_{y}\left(q_{x}\right)+d\left(p, p_{x}\right)=2$, which is independent of $x$. Of course, this is because $p$ is the focus of the parabola, $y=\frac{1}{4} x^{2}$. Therefore, we have

$$
b_{\gamma}\left(p_{x}\right)-b_{\gamma}\left(q_{x}\right) \geq \varepsilon,
$$

which is a contradiction since the left-hand side converges to zero as $x \rightarrow \infty$. Hence we conclude that the ray $\sigma$ is the vertical line in $R_{1}$.

If $\gamma$ is asymptotic to $\sigma$, then there is a sequence $\left\{t_{n}\right\}$ such that a minimal geodesic $\gamma_{n}$ from $q$ to $\sigma\left(t_{n}\right)$ intersects $\partial R_{2}$ at $q_{n}=\left(x_{n}, f\left(x_{n}\right)\right)$ and $x_{n} \rightarrow \infty$ as $n \rightarrow \infty$. By the same argument as before, we have

$$
\begin{aligned}
& b_{\sigma}(q)=\lim _{n \rightarrow \infty}\left(b_{\sigma}\left(p_{n}\right)+d\left(q_{n}, q\right)\right), \\
& b_{\sigma}(q) \leq b_{\sigma}\left(p_{0}\right)+d\left(p_{0}, q\right),
\end{aligned}
$$

where $p_{0}=(1,2,0) \in \partial R_{1}$. Since $b_{\sigma}\left(p_{0}\right)=0$ and $b_{\sigma}\left(p_{n}\right)=1-\frac{1}{4} x_{n}^{2}$, a simple computation gives

$$
d\left(p_{0}, q\right) \geq 2 \text {. }
$$

However, $d\left(p_{0}, q\right) \leq d\left(q_{0}, q\right)+\delta=1+\delta$, which is a contradiction since $\delta \leq \frac{1}{2}$. 
In this example, if $\sigma$ and $\gamma$ are vertical lines emanating from $(0,1,0) \in R_{1}$ and $(0,1, \alpha) \in R_{2}$ respectively, then $\sigma$ and $\gamma$ are asymptotic to each other. Suppose $b_{\sigma}-b_{\gamma} \equiv a$ along $\sigma$ and $b_{\sigma}-b_{\gamma} \equiv b$ along $\gamma$ for some constants $a$, $b$. Then we easily see that $a=-b \neq 0$. Hence this is also an example on which two rays asymptotic to each other do not define the same Busemann-function.

\section{REFERENCES}

1. W. Ballmann, M. Gromov, and V. Schroeder, Manifolds of nonpositive curvature, Progr. Math., Vol. 61, Birkhäuser, Boston, Basel, and Stuttgart, 1985.

2. J. Cheeger and D. Gromoll, Structure of complete manifolds of nonnegative curvature, Ann. of Math. (2) 96 (1972), 413-443.

3. S. Cohn-Vossen, Kürzeste Wege und Totalkrümmung auf Flächen, Compositio Math. 2 (1935), 63-133.

4. __ Totalkümmung und geodätischeLinien auf einfach zusammenhängenden offenenvolständigen Flächenstücken, Recueil Math. Moscow 43 (1936), 139-163.

5. J.-H. Eschenburg, Horospheres and the stable part of the geodesic flow, Math. Z. 153 (1977), 237-251.

6. M. Gromov, Hyperbolic manifolds, groups and actions, Riemann Surfaces and Related Topics, Stony Brook Conference, Ann. of Math. Stud., Vol. 97, Princeton Univ. Press, Princeton, NJ.

7. A. Kasue, A compactification of a manifold with asymptotically nonnegative curvature, Ann. Sci. École Norm. Sup. (4) 21 (1988), 593-622.

8. M. Maeda, A geometric significance of total curvature on complete open surfaces, Geometry of Geodesics and Related Topics, Adv. Stud. Pure Math., 3, Kinokuniya, Tokyo, 1984, pp. 451-458.

9. K. Shiohama, Topology of complete noncompact manifolds, Geometry of Geodesics and Related Topics, Adv. Stud. Pure Math., 3, Kinokuniya, Tokyo, 1984, pp. 423-450.

10. _ An integral formula for the measure of rays on complete open surfaces, J. Differential Geom. 94 (1986), 197-205.

11. T. Shioya, The ideal boundary of complete open surfaces, Tôhoku Math. J. 43 (1991), 37-59.

Department of Mathematics, Korea Advanced Institute of Science and Technology, TAEJON, KOREA

E-mail address: jwyim@math1.kaist.ac.kr 